\title{
NUEVA NORMATIVA DE TITULACIÓN EN EL RESIDENTADO MÉDICO EN EL PERÚ: PROBLEMAS Y PERSPECTIVAS
}

\author{
Rubén Valle ${ }^{1,2, a}$, Alberto Perales $3, b$
}

\begin{abstract}
RESUMEN
El 11 de mayo de 2015 salió publicado en el diario oficial "El Peruano" la modificación (RS 017-2015-SA) al Reglamento del Sistema Nacional de Residentado Médico (RS 002-2006-SA). Esta modificación establece que ya no es necesaria la presentación de una tesis o trabajo académico para obtener el título de médico especialista (artículo 28), pero sí realizar un "proyecto de trabajo" del área de su especialidad, que debe ser presentado al concluir el segundo año del residentado médico (Artículo 27). Esta noticia ha sido bien recibida por un sector de médicos residentes, mientras que otros consideran que la medida va en contra de su formación como especialistas. El presente artículo tiene por objetivo analizar la nueva normativa de titulación en el residentado médico en el Perú y sus implicancias para la investigación que se desarrolla durante esta etapa formativa de médicos especialistas.
\end{abstract}

Palabras clave: Tesis Académicas, Educación de Pregrado de Medicina, Internado y Residencia, Educación médica, (fuente: DeCS BIREME).

\section{NEW RULES FOR MEDICAL RESIDENCY QUALIFICATION IN PERU: PROBLEMS AND PERSPECTIVES}

\begin{abstract}
On May 11, 2015, amendment RS 017-2015-SA to the Regulation of the National System of Medical Residency (RS 002-2006-SA) was published in the Peruvian official newspaper "El Peruano". This amendment establishes that the submission of a thesis or scholarly work is not necessary to obtain the title of medical specialist (article 28), but when a "work project" is performed in a specialty area, it must be presented at the conclusion of the second year of medical residency (article 27). This news has been welcomed by one sector of medical residents, while others believe that the measure conflicts with their training as specialists. This article aims to analyze the new rules for medical residency qualification in Peru and its implications for research developed during this formative stage for specialist physicians.
\end{abstract}

Key words: Academic Dissertations; Education, Medical, Undergraduate; Internship and Residency; Education, Medical; (source: MeSH NLM).

\section{EL TRABAJO DE INVESTIGACIÓN ANTES DE LA EMISIÓN DE LA NUEVA NORMATIVA}

El programa de segunda especialidad profesional en Medicina Humana, denominado residentado médico, permite a los médicos cirujanos capacitarse en una especialidad y obtener el título de especialistas para su ejercicio profesional. La institución nacional responsable de la dirección de este programa es el Comité Nacional de Residentado Médico (CONAREME) creada por Decreto Supremo (DS) 055-75-SA en el año 1976 y, posteriormente, normada por el DS 00888-SA, Resolución Suprema (RS) 002-2006-SA y DS 010-2011-SA ${ }^{(1)}$. CONAREME trabaja en coordinación con instituciones del sector salud, instituciones representativas y universidades con facultades de Medicina en el Sistema Nacional del Residentado Médico (SINAREME) (2). Su objetivo es formar médicos especialistas de alta calidad científica y humanística, capaces de responder a las necesidades prioritarias de salud de la población mediante la estrecha interrelación de sus componentes ${ }^{(2)}$.

\footnotetext{
Instituto Nacional de Salud Mental Honorio Delgado-Hideyo Noguchi. Lima, Perú.

Facultad de Medicina. Universidad Nacional Mayor de San Marcos. Lima, Perú.

Instituto de Ética en Salud, Facultad de Medicina. Universidad Nacional Mayor de San Marcos. Lima, Perú.

Médico cirujano; ${ }^{\text {b }}$ médico psiquiatra

Recibido: 16/12/2015 Aprobado: 06/04/2016
}

Citar como: Valle R, Perales A. Nueva normativa de titulación en el residentado médico en el Perú: problemas y perspectivas. Rev Peru Med Exp Salud Publica. 2016;33(2):357-61. doi: 10.17843/rpmesp.2016.332.2142 
Hasta antes de mayo de 2015, el CONAREME regulaba la titulación de los médicos residentes en base al cumplimiento del artículo 28 del reglamento del sistema nacional deł residentado médico (RS 002-2006-SA) ${ }^{(3)}$. Según esta norma, para obtener el título de especialista, los médicos residentes debían aprobar los cursos lectivos de la especialidad y presentar a la universidad un trabajo de investigación. En ese sentido, los residentes debían preparar un proyecto de investigación, conducirlo y presentarlo a la universidad para su aprobación. Hasta donde hemos podido indagar, no se ha publicado ningún estudio que haya evaluado el desarrollo de estos trabajos en todo el programa del residentado médico. Solo una universidad peruana, que cuenta con una alta producción científica y con mecanismos de gestión de investigación bien establecidos, ha analizado el desarrollo de estos trabajos dentro de su programa, encontrando que el $64 \%$ de residentes tenían su proyecto de investigación aprobado por la universidad, y el $32,7 \%$ concluyó el trabajo de investigación ${ }^{(4)}$. Estos resultados no pueden ser generalizados a todo el residentado médico; sin embargo, muestran que hay un grupo de médicos residentes que no concluye su trabajo de investigación.

El incumplimiento del trabajo de investigación podría estar relacionado a la baja participación en investigación de los médicos durante el programa de residentado médico. Algunos estudios basados en muestras no probabilísticas encuentran que la mayoría de médicos residentes reporta no haber participado en investigación durante el residentado. Por ejemplo, en una encuesta aplicada a residentes de Medicina Interna de tres hospitales de Lima, el 80\% indicó que nunca había participado en investigación y $45 \%$ consideró como insuficiente la investigación en su sede ${ }^{(5)}$. Otro estudio realizado en egresados y residentes de los dos últimos años de la residencia de nefrología, encontró que el $67,5 \%$ nunca había participado en investigación, y entre aquellos que desarrollaron algún trabajo, ninguno lo llegó a publicar ${ }^{(6)}$. Una serie de barreras se han reconocido para el desarrollo de la investigación durante el residentado, como son el escaso número de tutores calificados ${ }^{(4,7)}$, ausencia o poco financiamiento de proyectos de investigación (4), y la alta carga laboral en el residentado médico ${ }^{(8)}$. Sin embargo, a pesar de estas dificultades, también se pueden observar trabajos de médicos residentes que son financiados por instituciones, publicados en revistas científicas, y difundidos en congresos. La baja participación en investigación, y específicamente la no ejecución del trabajo de investigación, ocasionó que muchos egresados del residentado médico no se hayan titulado y se encuentre ejerciendo la especialidad sin la debida certificación ${ }^{(4)}$.

\section{NUEVA NORMATIVA SOBRE LA TITULACIÓN EN EL RESIDENTADO MÉDICO}

A partir del 2013, la formación y regulación de la titulación de médicos especialistas adquirió mayor importancia debido a la implementación de la Reforma de Salud. La Reforma viene realizando una serie de cambios en el aseguramiento, equipamiento y mejoramiento de la infraestructura de los establecimientos de salud ${ }^{(9)}$. Sin embargo, una limitante para el cumplimiento de sus objetivos, es el déficit de médicos especialistas que existe en el Perú, el cual se calcula es del $45 \%{ }^{(10)}$. Ante este escenario, las instituciones encargadas de la formación de médicos especialistas determinaron incrementar las vacantes al residentado médico en las últimas convocatorias de admisión (11). Asimismo, la Asociación Peruana de Facultades de Medicina (ASPEFAM), en diciembre de 2013, dictó la norma de titulación de médicos especialistas por modalidad de evaluación de competencias. Mediante esta norma, los médicos egresados del programa de residentado médico que no se habían titulado, y que se encontraban ejerciendo la especialidad, podían titularse al acreditar experiencia en un servicio especializado por el tiempo de 3 años ${ }^{(12)}$. En ese sentido, la primera medida buscó aumentar el número de especialistas en el Perú, mientras que la segunda, regular la práctica de la medicina especializada.

Estas normativas, aunque bien orientadas, dejaban la posibilidad de formar un alto número de médicos especialistas, pero sin la debida titulación. Es decir, si los nuevos médicos residentes no cumplían con el requisito de ejecutar el trabajo de investigación se volvía al escenario de tener un alto número de egresados de especialidades no titulados. En este contexto, la Dirección General de Gestión del Desarrollo de Recursos Humanos del Ministerio de Salud (MINSA) sustentó la necesidad de realizar cambios al Reglamento del SINAREME (13). Las modificaciones se realizaron en base al numeral 45.3 del artículo 45 (Obtención de grados y títulos) de la Ley Universitaria 30220, y tuvo como objetivo adecuar la normativa del residentado médico a los desafíos de la Reforma de Salud ${ }^{(13,14)}$. Es así que el 11 de mayo de 2015 se publica en el "Diario Oficial El Peruano" la RS 017-2015-SA por la cual se realizan cambios al Reglamento Nacional del Residentado Médico (RS 002-2006-SA) ${ }^{(13)}$. La nueva normativa establece que para obtener el título de especialista en medicina ya no es necesaria la presentación de una tesis o trabajo académico (Artículo 28), pero sí realizar un proyecto de trabajo en el área de la especialidad, que debe ser presentado al concluir el segundo año de estudios (Artículo 27). Adicionalmente, permite que los médicos egresados del residentado médico que no cuenten con el título de especialistas puedan acogerse a la norma hasta un plazo máximo de 3 años (disposición complementaria transitoria) ${ }^{(13)}$.

En base a esta nueva normativa, el proyecto de trabajo en el área de la especialidad reemplaza a la tesis o trabajo académico, y será el nuevo requisito de titulación del residentado médico. Los médicos que se encuentren realizando el residentado deberán presentar su proyecto al concluir el segundo año de la residencia. 
Por otro lado, los médicos que egresaron del residentado médico, pero que no se titularon, deberán realizar los trámites necesarios de su titulación de haber cumplido con el requisito del proyecto de investigación.

\section{IMPLICANCIAS DE LA NUEVA NORMATIVA DE TITULACIÓN DEL RESIDENTADO MÉDICO}

El residentado médico abarca el periodo de capacitación del médico cirujano en una especialidad de la medicina. En sus inicios, la especialización se desarrollaba de forma no escolarizada y consistía en que el médico en formación trabajaba al lado de un "maestro" por un tiempo indefinido y sin un orden establecido en el entorno hospitalario de la especialidad ${ }^{(15)}$. Posteriormente, este sistema migró a la práctica escolarizada de la Medicina con la creación del residentado médico ${ }^{(1)}$. Desde su creación institucional, este programa ha tenido una serie de cambios que se han ido realizando con la finalidad de adaptar sus objetivos a las necesidades del sistema de salud y de fortalecer el programa a beneficio de la formación de los médicos especialistas. El más reciente cambio hecho al residentado médico es la derogación del trabajo de investigación como requisito para la titulación de especialista, restando de esta forma aspectos del campo de la investigación que eran considerados dentro del programa ${ }^{(13)}$.

La conducción de investigación durante el residentado médico ha mostrado tener beneficios, y oportunidades académicas para los médicos residentes (Tabla 1). Los residentes de los programas de Estados Unidos y Canadá reportan que realizar investigación durante el residentado trae como beneficios conocer un tema en profundidad, desarrollar habilidades analíticas (Ejm: estadística) ${ }^{(8)}$ e incrementar la satisfacción con la especialidad de entrenamiento ${ }^{(16)}$. Sin embargo, estos beneficios no son tangibles hasta que el residente se encuentra sumergido en el trabajo ${ }^{(8)}$. Agregado a ello, al finalizar los trabajos de investigación, se abren oportunidades de participar en conferencias científicas y de involucrarse en redes de investigación ${ }^{(8)}$, así como aumentar las probabilidades de obtener becas para estudios de posgrado más avanzados ${ }^{(17)}$. El desarrollo de investigación permite el aprendizaje de habilidades que de otra manera no se hubieran aprendido y que son de utilidad para el manejo clínico de pacientes tales como: búsqueda bibliográfica, análisis crítico, y el enfoque de medicina basada en la evidencia ${ }^{(18)}$. Finalmente, los residentes de Medicina que logran terminar sus trabajos de investigación describen la experiencia como una actividad que vale la pena realizar y terminan opinando que dicho trabajo debe ser un requerimiento del residentado médico ${ }^{(19)}$

A pesar de los potenciales beneficios que otorga el desarrollo de investigación, no todos están de acuerdo en que deba ser un requisito de los programas de residentado. Por ejemplo, en un estudio que encuestó a los residentes de ginecología y obstetricia en Canadá, se encontró que el $61 \%$ participaba en investigación solo porque era un requisito del programa ${ }^{(20)}$. Otro estudio, encontró que tanto residentes y profesores de un programa de residentado de psiquiatría en Estados Unidos consideraron a la investigación importante (98\%), pero solo $32 \%$ indicó que debería ser un requisito ${ }^{(21)}$. En Canadá, a pesar de estar normado que los programas de residentado médico soliciten el desarrollo de un trabajo académico a los residentes, solo el $73 \%$ de programas de residentado en psiquiatría solicita a sus residentes que desarrollen tal trabajo ${ }^{(22)}$. En Estados Unidos, una encuesta a médicos residentes de Medicina Interna encontró que solo el $69 \%$ consideraba que la investigación debería ser un requisito de la residencia ${ }^{(19)}$. En el Perú no existe información sobre este tema; sin embargo, la promulgación de la RS 017-2015-SA ha producido reacciones a favor y en contra de la medida. Mientras que un sector de médicos residentes ha recibido positivamente la normativa, otro sector considera que dicha medida va en contra de su formación como especialistas ${ }^{(23)}$.

La predisposición negativa al desarrollo de la tesis o trabajo académico podría deberse a la existencia de barreras que dificultan su ejecución durante el residentado médico (Tabla 2 ). Las dificultades más ampliamente reconocidas por los médicos de diferentes programas de residencia son las restricciones de tiempo y el escaso conocimiento de herramientas de investigación ${ }^{(19,20,24)}$. Por su propia estructura, el residentado médico es un programa principalmente clínico, con altas responsabilidades asistenciales en

Tabla 1. Beneficios del desarrollo de investigación durante el residentado médico

\footnotetext{
Beneficios

Alienta a un mejor manejo clínico de pacientes, fundamentado en la medicina basada en evidencia.

Desarrollo de habilidades de apreciación crítica de los resultados de investigación.

Contribuye al continuo crecimiento de la especialidad médica.

Mejora la satisfacción del residente con su especialidad.

Provee habilidades para un pensamiento crítico y de aprendizaje permanente.

Desarrollo de un conocimiento profundo sobre un tema de la especialidad.

Ofrece una perspectiva más amplia sobre los desafíos en el avance de la especialidad.

Genera satisfacción al haber contribuido en el avance de la especialidad.

Incrementa el número de investigadores clínicos.

Prestigio científico para el residente y para la institución patrocinadora.
} 
Tabla 2. Dificultades para el desarrollo de investigación durante el residentado médico

\begin{tabular}{l}
\hline Dificultades \\
\hline Restricciones de tiempo debido a deberes de la residencia. \\
Restricciones de tiempo por motivos personales. \\
Carencia de habilidades y conocimiento en estadística. \\
Escaso interés por parte de los profesores. \\
Falta de interés personal. \\
Ausencia de una relevante pregunta de investigación. \\
Problemas para reclutar pacientes. \\
Carencia de recursos para el desarrollo de investigación. \\
Escasa oferta de financiamiento. \\
\hline
\end{tabular}

donde las capacitaciones son preferentemente en el área de la especialidad y muy pocas o inexistentes en investigación ${ }^{(7,18,25)}$. En ese sentido, la estructura de muchos programas de residencia no facilita el desarrollo de investigación. En reconocimiento de estas debilidades, algunos programas han modificado su componente de investigación a través de diferentes estrategias, como la contratación de profesores con experiencia en investigación, habilitar recursos de investigación, e implementar rotaciones en unidades de investigación ${ }^{(18,24)}$. Los resultados de estas intervenciones muestran que los residentes incrementaron sustancialmente su participación en investigación y consideraron que la capacitación en investigación es esencial para la práctica de la medicina ${ }^{(18,24)}$. Por ende, es potencialmente posible incrementar la producción científica durante el residentado médico en base a la intervención en la propia estructura de los programas de residencia.

La residencia médica en el Perú solicitaba que cada uno de los egresados desarrolle una tesis o trabajo académico. La investigación científica no es algo que se pueda hacer por obligación, ni mucho menos cuando no se brindan las facilidades y capacitaciones para su desarrollo. En un escenario de tiempo restringido y de escasa capacitación en herramientas de investigación, el desarrollo de un trabajo de investigación podría resultar demandante para los residentes ${ }^{(26)}$. Aun en universidades extranjeras con alta producción científica, no se exige a todos los médicos residentes que desarrollen trabajos de investigación. En su lugar, se admite que los residentes puedan desarrollar otras formas de estudios como reporte de casos, revisión de temas clínicos y revisión de tópicos de investigación ${ }^{(22,24)}$. Si bien estos estudios no tienen las características de un trabajo de investigación, permiten cumplir con los requisitos de la parte académica, ser presentados en congresos y ser publicados en revistas científicas. En el Perú, el solicitar un trabajo de investigación como requisito de titulación mediante una Resolución Suprema normaba la ejecución de estos estudios; sin embargo, no fomentaba el desarrollo de los mismos. El fomento de la investigación corresponde a los programas de residentado promoviendo cambios en su estructura y brindando recursos para el desarrollo de los trabajos de investigación ${ }^{4,18,24)}$.

Actualmente, basados en la nueva normativa, los residentes deben presentar un proyecto de trabajo en el área de la especialidad que debe ser aprobado por la universidad aunque sin exigencia que sea desarrollado ${ }^{(13)}$. Un proyecto de trabajo es un documento en el cual se plasma las acciones a seguir para alcanzar un objetivo determinado, mas no representa un trabajo final del cual se obtenga un resultado. En ese sentido, la información no puede ser utilizada, a no ser que se ejecute, y para fines prácticos no representa ningún aporte para el desarrollo de la especialidad. La nueva normativa resulta extrema y restrictiva en relación al desarrollo de la investigación. Primero, no era imposible el desarrollo de un trabajo de investigación de forma curricular, y muestra de esto son los trabajos de los especialistas que han conseguido titularse por esta vía. En segundo lugar, al eliminar el trabajo de investigación, no se tomó en cuenta la opción de considerar otras formas de investigación como requisito de titulación, que aunque metodológicamente menos rigurosas, podrían contribuir al desarrollo de la especialidad. Finalmente, en forma de mecanismo de compensación por la eliminación del trabajo de investigación, tampoco se crearon canales para incentivarla por medio de mecanismos no curriculares por los cuales se reconozca el desarrollo de investigación realizada de forma voluntaria durante este periodo de entrenamiento.

La nueva norma favorece la titulación de los médicos egresados del residentado médico mas no favorece su capacitación, como se ha señalado. La norma resta requisitos a la titulación de médicos especialistas. Por lo tanto, no es una medida que incentive o promueva la capacitación en una habilidad adicional, sino que en buena medida, retira la investigación del proceso formativo de los médicos residentes. En ese sentido, la nueva normativa no representa ninguna contribución a la capacitación académica ni mucho menos científica de los médicos residentes.

Contribuciones de los autores: RV participó en la concepción del estudio, recolección de la información, análisis e interpretación de los datos y redacción del artículo. AP participó en la redacción del artículo, revisión crítica del artículo y en la aprobación de la versión final.

\section{Fuentes de financiamiento: autofinanciado}

Conflictos de interés: Los autores declaran que los comentarios y opiniones mencionados en el presente artículo son de su autoría y no necesariamente representa la posición de las instituciones donde laboran. 


\section{REFERENCIAS BIBLIOGRÁFICAS}

1. conareme.org.pe [internet]. Lima: Comité Nacional de Residentado Médico (CONAREME); [citado el 1 de noviembre de 2015]. Available from: http://www.conareme.org.pe/

2. Comité Nacional de Residentado Médico (CONAREME). Decreto supremo 008-88-SA. Normas básicas del sistema nacional del residentado médico. Lima: CONAREME; 1988.

3. Comité Nacional de Residentado Médico (CONAREME). Resolución Suprema 002-2006-SA. Reglamento del Sistema Nacional del Residentado Médico. Lima: CONAREME; 2006.

4. Ticse R, Pamo O, Samalvides F, Quispe T. Factores asociados a la culminación del proyecto de investigación requerido para optar el título de especialista en una Universidad peruana. Rev Peru Med Exp Salud Pública. 2014; 31(1):48-55.

5. Herrera-Añazco $P$, Hernández AV, Sánchez-Rivas F, Arana Maestre C. Percepción de la formación durante el residentado médico en hospitales del Ministerio de Salud de Lima, Perú. Rev Peru Med Exp Salud Pública. 2014;31(3):606-7.

6. Herrera-Anazco P, MezonesHolguin E, Hernandez AV. La investigación científica es deficiente entre residentes de nefrología: El caso peruano. Rev Nefrol Dialisis Transpl. 2014;34(3):159-61.

7. Ticse R, Ygreda P, Samalvides F. Publicación de los proyectos de investigación para la titulación como médicos especialistas de una Universidad peruana, 2007-2010. Rev Peru Med Exp Salud Pública. 2014;31(2):292-6.

8. Torous J, Padmanabhan J. Research by residents: obstacles and opportunities. Asian J Psychiatr. 2015;13:81-2. doi: 10.1016/j.ajp.2014.12.001.

9. Solari L. Reforma de salud en el Perú: Apuntando hacia la cobertura universal. Rev Peru Med Exp Salud Pública. 2014;31(4):623-5.

10. Zevallos L, Pastor R, Moscoso B. Oferta y demanda de médicos especialistas en los establecimientos de salud del Ministerio de Salud: Brechas a nivel nacional, por regiones y tipo de especialidad. Rev Peru Med Exp Salud Pública. 2011;28(2):177-85.

11. Universidad Nacional Mayor de San Marcos. Facultad de Medicina de "San Fernando". Prospecto Concurso de admisión al residentado médico 2014. Lima: UNMSM; 2014.

12. Asociación Peruana de Facultades de Medicina (ASPEFAM). Reglamento de titulación de médicos especialistas por modalidad de evaluación de competencias. Lima: ASPEFAM; 2013.

13. Aprueban la modificación al Reglamento del Sistema Nacional de Residentado Médico aprobado mediante Resolución Suprema № 002 2006-SA. Resolución Suprema 0172015-SA (11 de mayo 2015).

14. Ley Universitaria. Ley 30220 (9 de julio de 2014).

15. Nuñez U. El residentado médico en el Perú. Estado actual del residentado médico en el Perú. Simposio. Lima: Academia Nacional de Medicina Anales; 2005.

16. Takahashi O, Ohde S, Jacobs JL, Tokuda Y, Omata F, Fukui T. Residents' experience of scholarly activities is associated with higher satisfaction with residency training. J Gen Intern Med. 2009;24(6):716-20. doi: 10.1007/ s11606-009-0970-4.

17. Souba WW, Tanabe KK, Gadd MA, Smith BL, Bushman MS. Attitudes and opinions toward surgical research. A survey of surgical residents and their chairpersons. Ann Surg. 1996; 223(4):377-83.

18. Kanna B, Deng C, Erickson SN, Valerio JA, Dimitrov V, Soni A. The research rotation: competency-based structured and novel approach to research training of internal medicine residents. BMC Med Educ. 2006;6:52.

19. Rivera JA, Levine RB, Wright SM. Completing a scholarly project during residency training. Perspectives of residents who have been successful. J
Gen Intern Med. 2005;20(4):366-9.

20. Clancy AA, Posner G. Attitudes Toward Research During Residency: A Survey of Canadian Residents in Obstetrics and Gynecology. J Surg Educ. 2015;72(5):836-43. doi: 10.1016/j.jsurg.2015.02.007.

21. Fitz-Gerald M, Kablinger A, Manno B, Carter O, Caldito G, Smith S. Psychiatry Residents' Participation in Research. A survey of attitudes and experiences. Academic Psychiatry. 2001;25(1):42-6.

22. Shanmugalingam A, Ferreria SG, Norman RM, Vasudev K. Research experience in psychiatry residency programs across Canada: current status. Can J Psychiatry. 2014; 59(11):58690.

23. Asociación Nacional de Médicos Residentes del Perú. Informe 003-2015-ANMRP. Sobre las modificaciones al reglamento del Sistema Nacional del Residentado Médico. Lima: ANMRP; 2015.

24. Levine RB, Hebert RS, Wright SM. Resident research and scholarly activity in internal medicine residency training programs. J Gen Intern Med. 2005;20(2):155-9.

25. Herrera-Añazco P, Galán-Rodas E, Mezones-Holguín E. Residentado Médico en el Perú: Una visión más allá de la demanda. Acta Med Per. 2012;29(1):10-1.

26. Comité Nacional de Residentado Médico (CONAREME). Seminario Internacional 25 Aniversario del Sistema Nacional del Residentado Médico. Taller Internacional "Experiencias Internacionales en la formación de médicos especialistas. Lima, Perú, 26y 27 de septiembre de 2001. Lima: CONAREME; 2002.

Correspondencia: Rubén Valle

Dirección: Calle Octavio Bernal 588 Dpto 402 Jesus María. Lima, Perú.

Teléfono: (511) 985835737

Correo electrónico: ruben_vr12@hotmail.com 